\title{
EXTENSION OF THE PUFEM TO ELASTIC WAVE PROPAGATION IN LAYERED MEDIA
}

\author{
O. Laghrouche ${ }^{1}$, A. El Kacimi ${ }^{1}$ and J. Trevelyan ${ }^{2}$ \\ ${ }^{1}$ School of the Built Environment \\ Heriot-Watt University, Edinburgh EH14 4AS, UK \\ e-mail: o.laghrouche@hw.ac.uk, a.elkacimi@hw.ac.uk \\ ${ }^{2}$ School of Engineering and Computing Sciences \\ Durham University, Durham DH1 3LE, UK \\ e-mail: jon.trevelyan@durham.ac.uk
}

Keywords: Elastic waves, finite elements, plane wave basis, Lagrange multipliers, layered media, free surface, Rayleigh waves.

\begin{abstract}
This work deals with the extension of the Partition of Unity Finite Element Method (PUFEM) to solve wave problems involving propagation, transmission and reflection in layered elastic media. Problems dealing with wave reflection at a free surface and propagation of pure Rayleigh waves are also considered. The proposed method consist to apply the plane wave basis decomposition to the elastic wave equation in each layer of the elastic medium and then enforce necessary continuity conditions at the interfaces through the use of Lagrange multipliers. The accuracy and effectiveness of the proposed technique is determined by comparing results for selected problems with known analytical solutions.
\end{abstract}




\section{INTRODUCTION}

Plane wave basis finite elements capable of containing many wavelengths per nodal spacing were developed and implemented to solve wave scattering problems in homogeneous elastic media $[1,2,3]$. These elements allow us to relax the traditional requirement of around ten nodal points per wavelength and therefore lead to huge savings in computing effort. Many other techniques were developed for the same objective, such as the Discontinuous Enrichment Method [4], the Ultra Weak Variational Formulation [5] and the Partition of Unity Boundary Element Method [6], for example.

In this work, problems of practical interest such as those encountered in soil wave propagation and scattering are considered. For a layered elastic medium, the displacement field is expressed as a combination of propagating planar pressure and shear waves within each homogeneous layer. At the interface between two layers, necessary continuity conditions are enforced through the use of Lagrange multipliers, which are also approximated using the plane wave decomposition approach [7].

After presenting relevant theory, some preliminary numerical tests are carried out. The first problem deals with elastic waves propagating in a homogeneous medium and hitting a plane free surface, for angles of incidence above and below the critical angle. The second test problem concerns the propagation of pure Rayleigh waves, which are known to propagate with a specific wave number different from the pressure and shear wave numbers. Last, reflection and transmission of elastic waves at a plane interface between two elastic media will be considered. For the first two problems, there is no need to incorporate the Lagrange multipliers as no inhomogeneity is involved in the problems unlike in the third one where they are necessary to ensure compatibility conditions at the interface.

\section{MATHEMATICAL FORMULATIONS}

Let $\Omega$ be a space domain in $\mathbb{R}^{2}$ occupied by an elastic inhomogeneous medium whose elastic properties are piecewise constant such as, for example, a multilayered soil medium where each layer is homogenous. It is then adequate to assume that the domain $\Omega$ is composed of multiple homogeneous linear and isotropic subdomains $\Omega_{j}$ with the Lamé coefficients $\lambda_{j}$ and $\mu_{j}$, and density $\rho_{j}$. In this paper, for simplicity, the two-subdomain problem shown in Figure 1 is considered where $\Omega=\Omega_{1} \cup \Omega_{2}$ bounded by $\Gamma=\Gamma_{1} \cup \Gamma_{2}$ with the boundary $\Gamma_{\text {int }}$ being the interface between $\Omega_{1}$ and $\Omega_{2}$. We will denote by $(\mathbf{i}, \mathbf{j})$ the cartesian vector system and by $\mathbf{x}=x \mathbf{i}+y \mathbf{j}$ a generic point in $\mathbb{R}^{2}$. We will also assume a harmonic steady state problem and hence the time variable is omitted in the formulation.

\subsection{Problem formulation}

Let us first consider the subdomain $\Omega_{1}$ bounded by $\Gamma_{1} \cup \Gamma_{\text {int }}$. The time independent displacement field $\mathbf{u}_{1}$ satisfies the Navier's equation

$$
-\rho_{1} \omega^{2} \mathbf{u}_{1}-\nabla \cdot \boldsymbol{\sigma}\left(\mathbf{u}_{1}\right)=\rho_{1} \mathbf{f}_{1}
$$

where $\omega$ is the circular frequency, $\rho_{1}$ is the density and $\mathbf{f}_{\mathbf{1}}$ is the body force. The stress tensor $\boldsymbol{\sigma}$ is defined via the classical Hooke's law

$$
\boldsymbol{\sigma}\left(\mathbf{u}_{1}\right)=\lambda_{1} \nabla \cdot \mathbf{u}_{1} \mathbf{I}+\mu_{1}\left(\nabla \mathbf{u}_{1}+\nabla \mathbf{u}_{1}^{\top}\right),
$$

where I denotes the identity matrix, $\lambda_{1}$ and $\mu_{1}$ are the Lamé coefficients, and $\nabla \mathbf{u}_{1}=\left(\nabla u_{1}, \nabla v_{1}\right)^{\top}$, with $u_{1}$ and $v_{1}$ being the horizontal and vertical components of the displacement field $\mathbf{u}_{\mathbf{1}}$. The 
notation ' $T$ ' stands for the transpose of a given vector or tensor. The dot product ' '' of $\nabla$ and the tensor field $\boldsymbol{\sigma}=\left(\boldsymbol{\sigma}_{x}, \boldsymbol{\sigma}_{y}\right)^{\top}$ in $\mathbb{C}^{2} \times \mathbb{C}^{2}$ is defined by

$$
\nabla \cdot \boldsymbol{\sigma}=\left(\begin{array}{c}
\nabla \cdot \boldsymbol{\sigma}_{x} \\
\nabla \cdot \boldsymbol{\sigma}_{y}
\end{array}\right)
$$

with $\nabla$ being the gradient operator. In addition to expressions (1) and (2), the formulation of the problem is completed by the boundary conditions imposed on $\Gamma_{1}$. Here we consider a Robin type boundary conditions such that

$$
\boldsymbol{\sigma}\left(\mathbf{u}_{1}\right) \mathbf{n}_{1}=\mathrm{i}\left[\left(\lambda_{1}+2 \mu_{1}\right) k_{1}^{P}\left(\mathbf{u}_{1} \cdot \mathbf{n}_{1}\right) \mathbf{n}_{1}+\mu_{1} k_{1}^{S}\left(\mathbf{u}_{1} \cdot \mathbf{t}_{1}\right) \mathbf{t}_{1}\right]+\mathbf{g}_{1} .
$$

In the above expression, $i$ represents the imaginary number such that $i^{2}=-1, \mathbf{n}_{\mathbf{1}}$ and $\mathbf{t}_{\mathbf{1}}$ are

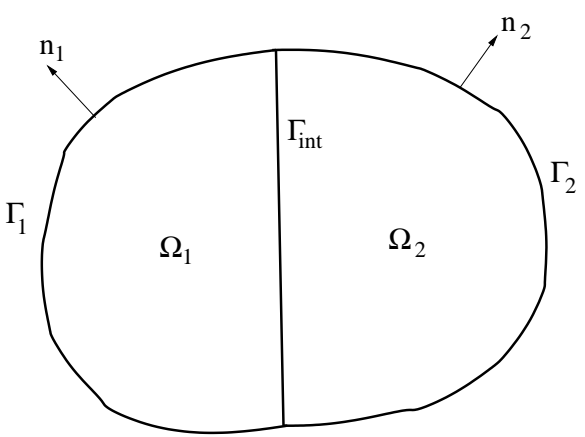

Figure 1: A two-subdomain example.

the normal and tangential vectors to the boundary. The wave numbers $k_{1}^{P}$ and $k_{1}^{S}$ correspond to the pressure $(\mathrm{P})$ and shear $(\mathrm{S})$ waves, respectively. They are defined by

$$
k_{1}^{P}=\frac{\omega}{c_{1}^{P}} \quad \text { and } \quad k_{1}^{S}=\frac{\omega}{c_{1}^{S}} .
$$

The P-wave and S-wave speeds are given by

$$
c_{1}^{P}=\left(\frac{\lambda_{1}+2 \mu_{1}}{\rho_{1}}\right)^{\frac{1}{2}} \quad \text { and } \quad c_{1}^{S}=\left(\frac{\mu_{1}}{\rho_{1}}\right)^{\frac{1}{2}} .
$$

In expression 4 , the source term $\mathbf{g}_{1}$ will involve analytical solutions of elastic wave problems. This approach is adopted with the aim to validate the proposed method and assess its performance. But in practice absorbing boundary conditions would rather be used and would therefore involve extra numerical errors.

\subsection{Weighted residual scheme}

Let us multiply equation (1) by the complex conjugate of a test function $\mathbf{w}_{1}$

$$
\int_{\Omega_{1}}\left(-\rho_{1} \omega^{2} \mathbf{u}_{1}-\nabla \cdot \boldsymbol{\sigma}\left(\mathbf{u}_{1}\right)\right) \cdot \overline{\mathbf{w}}_{1} d \Omega=\int_{\Omega_{1}} \rho_{1} \mathbf{f}_{1} \cdot \overline{\mathbf{w}}_{1} d \Omega
$$

Integrating by parts over $\Omega_{1}$, the following weak form is obtained

$$
\begin{aligned}
& -\omega^{2} \rho_{1} \int_{\Omega_{1}} \mathbf{u}_{1} \cdot \overline{\mathbf{w}}_{1} d \Omega-\int_{\Gamma_{1}} \boldsymbol{\sigma}\left(\mathbf{u}_{1}\right) \mathbf{n}_{1} \cdot \overline{\mathbf{w}}_{1} d \Gamma-\int_{\Gamma_{i n t}} \boldsymbol{\sigma}\left(\mathbf{u}_{1}\right) \mathbf{n}_{1} \cdot \overline{\mathbf{w}}_{1} d \Gamma+\int_{\Omega_{1}} \boldsymbol{\sigma}\left(\mathbf{u}_{1}\right) \cdot \nabla \overline{\mathbf{w}}_{1} d \Omega \\
& =\rho_{1} \int_{\Omega_{1}} \mathbf{f}_{1} \cdot \overline{\mathbf{w}}_{1} d \Omega .
\end{aligned}
$$


For practicality let us express the stress tensor in terms of the displacement field by using the following expression

$$
\boldsymbol{\sigma}\left(\mathbf{u}_{1}\right)=\lambda_{1} \nabla \cdot \mathbf{u}_{1} \mathbf{I}+\mu_{1} \nabla \times \mathbf{u}_{1} \mathbf{J}+2 \mu_{1} \nabla \mathbf{u}_{1}
$$

where the matrix $\mathbf{J}$ is defined by

$$
\mathbf{J}=\left(\begin{array}{cc}
0 & 1 \\
-1 & 0
\end{array}\right)
$$

and also re-write $\boldsymbol{\sigma}\left(\mathbf{u}_{\mathbf{1}}\right) \cdot \nabla \overline{\mathbf{w}}_{1}$ in expression (8) under the following form

$$
\boldsymbol{\sigma}\left(\mathbf{u}_{1}\right) \cdot \nabla \overline{\mathbf{w}}_{1}=\lambda_{1}\left(\nabla \cdot \mathbf{u}_{1}\right)\left(\nabla \cdot \overline{\mathbf{w}}_{1}\right)+2 \mu_{1} \nabla \mathbf{u}_{1} \cdot \nabla \overline{\mathbf{w}}_{1}-\mu_{1}\left(\nabla \times \mathbf{u}_{1}\right)\left(\nabla \times \overline{\mathbf{w}}_{1}\right) .
$$

Now using the boundary condition (4), the above variational formulation becomes

$$
\begin{aligned}
& -\omega^{2} \rho_{1} \int_{\Omega_{1}} \mathbf{u}_{1} \cdot \overline{\mathbf{w}}_{1} d \Omega+2 \mu_{1} \int_{\Omega_{1}} \nabla \mathbf{u}_{1} \cdot \nabla \overline{\mathbf{w}}_{1} d \Omega-\mu_{1} \int_{\Omega_{1}}\left(\nabla \times \mathbf{u}_{1}\right)\left(\nabla \times \overline{\mathbf{w}}_{1}\right) d \Omega \\
& +\lambda_{1} \int_{\Omega_{1}}\left(\nabla \cdot \mathbf{u}_{1}\right)\left(\nabla \cdot \overline{\mathbf{w}}_{1}\right) d \Omega-\mathrm{i} \int_{\Gamma_{1}}\left[\left(\lambda_{1}+2 \mu_{1}\right) k_{1}^{P}\left(\mathbf{u}_{1} \cdot \mathbf{n}_{1}\right)\left(\overline{\mathbf{w}}_{1} \cdot \mathbf{n}_{1}\right)+\mu_{1} k_{1}^{S}\left(\mathbf{u}_{1} \cdot \mathbf{t}_{1}\right)\left(\overline{\mathbf{w}}_{1} \cdot \mathbf{t}_{1}\right)\right] d \Gamma \\
& -\int_{\Gamma_{\text {int }}} \boldsymbol{\sigma}\left(\mathbf{u}_{1}\right) \mathbf{n}_{1} \cdot \overline{\mathbf{w}}_{1} d \Gamma=\rho_{1} \int_{\Omega_{1}} \mathbf{f} \cdot \overline{\mathbf{w}}_{1} d \Omega+\int_{\Gamma_{1}} \mathbf{g}_{1} \cdot \overline{\mathbf{w}}_{1} d \Gamma .
\end{aligned}
$$

Following the same approach, a similar weak form is obtained for the subdomain $\Omega_{2}$ bounded by $\Gamma_{2} \cup \Gamma_{\text {int }}$ such that

$$
\begin{aligned}
& -\omega^{2} \rho_{2} \int_{\Omega_{2}} \mathbf{u}_{2} \cdot \overline{\mathbf{w}}_{2} d \Omega+2 \mu_{2} \int_{\Omega_{2}} \nabla \mathbf{u}_{2} \cdot \nabla \overline{\mathbf{w}}_{2} d \Omega-\mu_{2} \int_{\Omega_{2}}\left(\nabla \times \mathbf{u}_{2}\right)\left(\nabla \times \overline{\mathbf{w}}_{2}\right) d \Omega \\
& +\lambda \int_{\Omega_{2}}\left(\nabla \cdot \mathbf{u}_{2}\right)\left(\nabla \cdot \overline{\mathbf{w}}_{2}\right) d \Omega-\mathrm{i} \int_{\Gamma_{2}}\left[\left(\lambda_{2}+2 \mu_{2}\right) k_{2}^{P}\left(\mathbf{u}_{2} \cdot \mathbf{n}_{2}\right)\left(\overline{\mathbf{w}}_{2} \cdot \mathbf{n}_{2}\right)+\mu_{2} k_{2}^{S}\left(\mathbf{u}_{2} \cdot \mathbf{t}_{2}\right)\left(\overline{\mathbf{w}}_{2} \cdot \mathbf{t}_{2}\right)\right] d \Gamma \\
& -\int_{\Gamma_{\text {int }}} \boldsymbol{\sigma}\left(\mathbf{u}_{2}\right) \mathbf{n}_{2} \cdot \overline{\mathbf{w}}_{2} d \Gamma=\rho_{2} \int_{\Omega_{2}} \mathbf{f} \cdot \overline{\mathbf{w}}_{2} d \Omega+\int_{\Gamma_{2}} \mathbf{g}_{2} \cdot \overline{\mathbf{w}}_{2} d \Gamma,
\end{aligned}
$$

where all functions and parameters are defined in a similar way as for the weak form (12) replacing the subscript 1 by 2 .

\subsection{Compatibility conditions}

At the interface $\Gamma_{\text {int }}$, between $\Omega_{1}$ and $\Omega_{2}$, continuity of displacements and stresses must be satisfied

$$
\begin{gathered}
\mathbf{u}_{1}=\mathbf{u}_{2}, \\
\boldsymbol{\sigma}\left(\mathbf{u}_{1}\right) \mathbf{n}_{1}=-\boldsymbol{\sigma}\left(\mathbf{u}_{2}\right) \mathbf{n}_{2} .
\end{gathered}
$$

Let us introduce the Lagrange multiplier $\nu$ defined by

$$
\boldsymbol{\nu}=\boldsymbol{\sigma}\left(\mathbf{u}_{1}\right) \mathbf{n}_{1}=-\boldsymbol{\sigma}\left(\mathbf{u}_{2}\right) \mathbf{n}_{2}
$$


The weak forms (12) and (13) become then

$$
\begin{aligned}
& -\omega^{2} \rho_{1} \int_{\Omega_{1}} \mathbf{u}_{1} \cdot \overline{\mathbf{w}}_{1} d \Omega+2 \mu_{1} \int_{\Omega_{1}} \nabla \mathbf{u}_{1} \cdot \nabla \overline{\mathbf{w}}_{1} d \Omega-\mu_{1} \int_{\Omega_{1}}\left(\nabla \times \mathbf{u}_{1}\right)\left(\nabla \times \overline{\mathbf{w}}_{1}\right) d \Omega \\
& +\lambda_{1} \int_{\Omega_{1}}\left(\nabla \cdot \mathbf{u}_{1}\right)\left(\nabla \cdot \overline{\mathbf{w}}_{1}\right) d \Omega-\mathrm{i} \int_{\Gamma_{1}}\left[\left(\lambda_{1}+2 \mu_{1}\right) k_{1}^{P}\left(\mathbf{u}_{1} \cdot \mathbf{n}_{1}\right)\left(\overline{\mathbf{w}}_{1} \cdot \mathbf{n}_{1}\right)+\mu_{1} k_{1}^{S}\left(\mathbf{u}_{1} \cdot \mathbf{t}_{1}\right)\left(\overline{\mathbf{w}}_{1} \cdot \mathbf{t}_{1}\right)\right] d \Gamma \\
& -\int_{\Gamma_{\text {int }}} \boldsymbol{\nu} \cdot \overline{\mathbf{w}}_{1} d \Gamma=\rho_{1} \int_{\Omega_{1}} \mathbf{f} \cdot \overline{\mathbf{w}}_{1} d \Omega+\int_{\Gamma_{1}} \mathbf{g}_{1} \cdot \overline{\mathbf{w}}_{1} d \Gamma
\end{aligned}
$$

and

$$
\begin{aligned}
& -\omega^{2} \rho_{2} \int_{\Omega_{2}} \mathbf{u}_{2} \cdot \overline{\mathbf{w}}_{2} d \Omega+2 \mu_{2} \int_{\Omega_{2}} \nabla \mathbf{u}_{2} \cdot \nabla \overline{\mathbf{w}}_{2} d \Omega-\mu_{2} \int_{\Omega_{2}}\left(\nabla \times \mathbf{u}_{2}\right)\left(\nabla \times \overline{\mathbf{w}}_{2}\right) d \Omega \\
& +\lambda_{2} \int_{\Omega_{2}}\left(\nabla \cdot \mathbf{u}_{2}\right)\left(\nabla \cdot \overline{\mathbf{w}}_{2}\right) d \Omega-\mathrm{i} \int_{\Gamma_{2}}\left[\left(\lambda_{2}+2 \mu_{2}\right) k_{P}\left(\mathbf{u}_{2} \cdot \mathbf{n}_{2}\right)\left(\overline{\mathbf{w}}_{2} \cdot \mathbf{n}_{2}\right)+\mu_{2} k_{S}\left(\mathbf{u}_{2} \cdot \mathbf{t}_{2}\right)\left(\overline{\mathbf{w}}_{2} \cdot \mathbf{t}_{2}\right)\right] d \Gamma \\
& +\int_{\Gamma_{\text {int }}} \boldsymbol{\nu} \cdot \overline{\mathbf{w}}_{2} d \Gamma=\rho_{2} \int_{\Omega_{2}} \mathbf{f} \cdot \overline{\mathbf{w}}_{2} d \Omega+\int_{\Gamma_{2}} \mathbf{g}_{2} \cdot \overline{\mathbf{w}}_{2} d \Gamma .
\end{aligned}
$$

It is clear from the above weak forms, (17) and (18), that the problem is augmented as the Lagrange multiplier $\boldsymbol{\nu}$ is introduced as an extra unknown in the problem. The integration of the two weak forms leads to a rectangular system of equations, where the number of unknowns exceeds the number of equations. Extra equations are therefore added by ensuring the continuity of displacements such as in expression (14). This is done through the weak form

$$
\int_{\Gamma_{i n t}}\left(\overline{\mathbf{u}_{1}}-\overline{\mathbf{u}_{2}}\right) \cdot \boldsymbol{\delta} d \Gamma=0
$$

where $\delta$ is a test function in the space of the Lagrange multipliers.

In the following subsection both the displacement field and the Lagrange multiplier will be approximated via plane wave basis finite elements.

\subsection{Plane wave approximation}

The computational domain $\Omega$ is meshed into finite elements with $n$ nodal points in total. We denote by $N^{z}, z=1, n$, the partition of unity by polynomial finite element shape functions, and respectively by $m_{p}$ and $m_{s}$ the number of approximating $\mathrm{P}$ and $\mathrm{S}$ plane waves. The displacement $\mathbf{u}_{j}$ in a subdomain $\Omega_{j}$ is approximated as follows $[1,2]$

$$
\mathbf{u}_{j}=\sum_{z=1}^{n} \sum_{l=1}^{m_{P}} N^{z} A_{z, l}^{P} \exp \left(\mathrm{i} k_{j}^{P} \mathbf{x} \cdot \mathbf{d}_{P}^{l}\right) \mathbf{d}_{P}^{l}+\sum_{z=1}^{n} \sum_{l=1}^{m_{S}} N^{z} A_{z, l}^{S} \exp \left(\mathrm{i} k_{j}^{S} \mathbf{x} \cdot \mathbf{d}_{S}^{l}\right) \mathbf{d}_{S, \perp}^{l} .
$$

The amplitudes $A_{z, l}^{P}$ and $A_{z, l}^{S}$, at a given node $z$ and corresponding to $\mathrm{P}$ and $\mathrm{S}$ plane waves travelling in the directions $\mathbf{d}_{P}^{l}$ and $\mathbf{d}_{S}^{l}$, respectively, are now the unknowns of the problem. The orthogonal of a vector $\mathbf{d}$ denoted by $\mathbf{d}_{\perp}$ is defined by $\mathbf{d}_{\perp}=-\mathbf{J d}$, where the rotation matrix $\mathbf{J}$, with the angle $\frac{\pi}{2}$, is given in expression (10). The directions $\mathbf{d}_{P}^{l}$ and $\mathbf{d}_{S}^{l}$ are taken uniformly distributed on the unit circle such that

$$
\begin{array}{ll}
\mathbf{d}_{P}^{l}=\left(\cos \theta_{P}^{l}, \sin \theta_{P}^{l}\right)^{\top}, & \theta_{P}^{l}=\frac{2 \pi l}{m_{P}} \\
\mathbf{d}_{S}^{l}=\left(\cos \theta_{S}^{l}, \sin \theta_{S}^{l} p\right)^{\top}, & \theta_{S}^{l}=\frac{2 \pi l}{m_{S}} .
\end{array}
$$


It is obvious from the above expression (20) of the displacement field that the pair of wave numbers $\left(k_{j}^{P}, k_{j}^{S}\right)$ will take different values depending to which subdomain, $\Omega_{1}$ or $\Omega_{2}$, the considered finite element belongs to.

For the approximation of the Lagrange multiplier $\nu$, the approach is inspired from previous work [7], which consists to write

$$
\boldsymbol{\nu}=\sum_{z=1}^{n} \sum_{l=1}^{m_{P}} N^{z} B_{z, l}^{P} \exp \left(\mathrm{i} k^{P} \mathbf{x} \cdot \mathbf{d}_{P}^{l}\right) \mathbf{d}_{P}^{l}+\sum_{z=1}^{n} \sum_{l=1}^{m_{S}} N^{z} B_{z, l}^{S} \exp \left(\mathrm{i} k^{S} \mathbf{x} \cdot \mathbf{d}_{S}^{l}\right) \mathbf{d}_{S, \perp}^{l} .
$$

In other words, the lagrange multiplier is also approximated by sets of $\mathrm{P}$ and $\mathrm{S}$ plane waves, and the unknowns $B_{z, l}^{P}$ and $B_{z, l}^{S}$ are in a sense the amplitudes of the approximating plane waves. At the interface between $\Omega_{1}$ and $\Omega_{2}$, the pair of wave numbers $\left(k^{P}, k^{S}\right)$ is chosen, for example, such that

$$
k^{P}=\max \left(k_{1}^{P}, k_{2}^{P}\right) \quad \text { and } \quad k^{S}=\max \left(k_{1}^{S}, k_{2}^{S}\right)
$$

The numbers $m_{P}$ and $m_{S}$ of approximating plane waves may be constant or may vary depending whether the approximation is applied to the displacements fields $\mathbf{u}_{\mathbf{1}}$ and $\mathbf{u}_{\mathbf{2}}$ or to the Lagrange multiplier $\boldsymbol{\nu}$. Expressions (17), (18) and (19) lead to square system matrix, where the solution is obtained through a direct solver which uses a factorization of the form $\mathbf{L D L} \mathbf{L}^{H}$ where $\mathbf{D}$ is a real diagonal matrix, $\mathbf{L}$ is a lower triangular matrix with diagonal entries equal to 1 and $\mathbf{L}^{H}$ is the transpose conjugate of $\mathbf{L}$ [8]. It is worth mentioning that the element matrices are obtained via an exact integration developed in reference [2] and hence the computations are significantly faster compared to past work [1] where high order Gauss-Legendre quadrature schemes were used involving thousands of integration points per finite element.

In the next section, some preliminary numerical results validating the proposed model are presented.

\section{PRELIMINARY RESULTS}

The model presented above is validated by solving typical problems of practical interest such as those encountered in soil wave propagation, which involve interfaces between the soil layers and a free surface. First, SV elastic waves propagating in a homogeneous medium and hitting a plane free surface is considered. Then, a problem with pure Rayleigh waves is dealt with, where the displacement field approximation involves $\mathrm{P}$ and $\mathrm{S}$ plane waves, rather than plane waves with the Rayleigh wave number. Last, a test problem involving reflection and transmission of elastic waves at a plane interface between two semi-infinite elastic media is carried out. All considered problems have analytical solutions presented in various textbooks, such as reference [9]. To assess the accuracy of the numerical solution in percentage the following $L^{2}$ error

$$
\varepsilon_{2}=\frac{\|\mathbf{u}-\widetilde{\mathbf{u}}\|_{L_{2}(\Omega)}}{\|\widetilde{\mathbf{u}}\|_{L_{2}(\Omega)}} \times 100 \%
$$




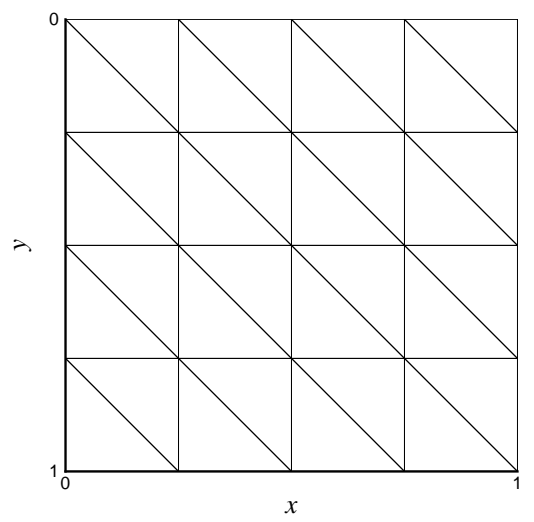

Figure 2: Mesh of the computational domain.

is used where $\mathbf{u}$ is the numerical solution and $\widetilde{\mathbf{u}}$ is the analytical solution of the considered problem. The chosen computational domain is a square of unit length. It is meshed into 3-node triangular finite elements with 32 elements and 25 nodes (Figure 2).

\subsection{SV plane wave hitting a free surface}

This is a common situation occurring at the soil free surface, for example. Let us consider a shear wave, SV, of unit amplitude incident with an angle $\beta$ (Figure 3). In general, this leads to both reflected compression and shear waves. The compression wave potential is therefore given only by the reflected wave

$$
\phi=R_{P} e^{\mathrm{i} k(-x-y \tan \alpha)},
$$

and the shear wave potential is the sum of the incident and the reflected waves

$$
\psi=1 e^{\mathrm{i} k(-x+y \tan \beta)}+R_{S} e^{\mathrm{i} k(-x-y \tan \beta)},
$$

where $k$ is the wave number such that $k=k_{P} \cos \alpha=k_{S} \cos \beta$, with $\alpha$ being the angle of the reflected P-wave. The reflection coefficients $R_{P}$ and $R_{S}$ are computed by ensuring zero-stress boundary conditions at the free surface [9]. The displacements are computed via the following derivatives of the potentials

$$
u=\frac{\partial \phi}{\partial x}-\frac{\partial \psi}{\partial y} \quad \text { and } \quad v=\frac{\partial \phi}{\partial y}+\frac{\partial \psi}{\partial x}
$$

Snell's law states that $c_{P} / \cos \alpha=c_{S} / \cos \beta$ and hence $\tan \alpha=\left(1 / 3 \cos ^{2} \beta-1\right)^{1 / 2}$ for this elastic medium, with $\lambda=\mu=\rho=1$. Consequently, the reflected compression wave exists only if the angle of incidence $\beta$ reaches the critical angle $\beta_{c}=\cos ^{-1}(1 / \sqrt{3})$ and that for all angles $0<\beta<\cos ^{-1}(1 / \sqrt{3})$ there is no reflected compression wave. In this latter case, $\tan \alpha$ becomes a complex number and the motion corresponding to the $\mathrm{P}$-wave potential propagates along the free surface, as an edge wave, and decreases exponentially with depth, an an evanescent mode. 


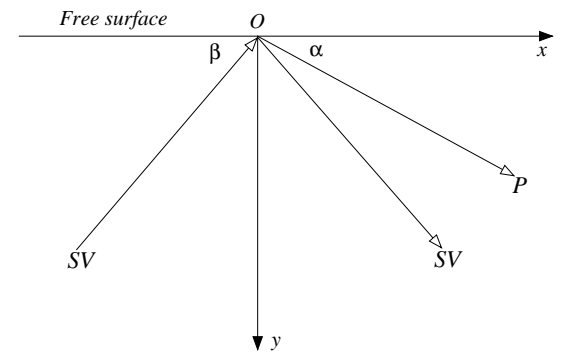

Figure 3: SV-plane wave hitting a free surface of a semi-infinite elastic medium.

Figure 7 shows, for $\omega=30$, contour plots of the real part of the displacement for two cases of incidence. In the first case (left), the angle of incidence $\beta<\beta_{c}$, which means both $\mathrm{P}$ and $\mathrm{S}$ waves are reflected. In the second one, $\beta>\beta_{c}$ and hence only an $\mathrm{S}$ wave is reflected while there is an edge P-wave wave decaying exponentially with depth. In both cases, a total of 32 plane waves are used in the approximation of the displacement field $\mathbf{u}$, with $m_{P}=m_{S}=16$. The $L_{2}$ errors, in both cases $\varepsilon_{2}=0.6 \%$, show that the numerical solution is good agreement with the analytical solution of the problem.
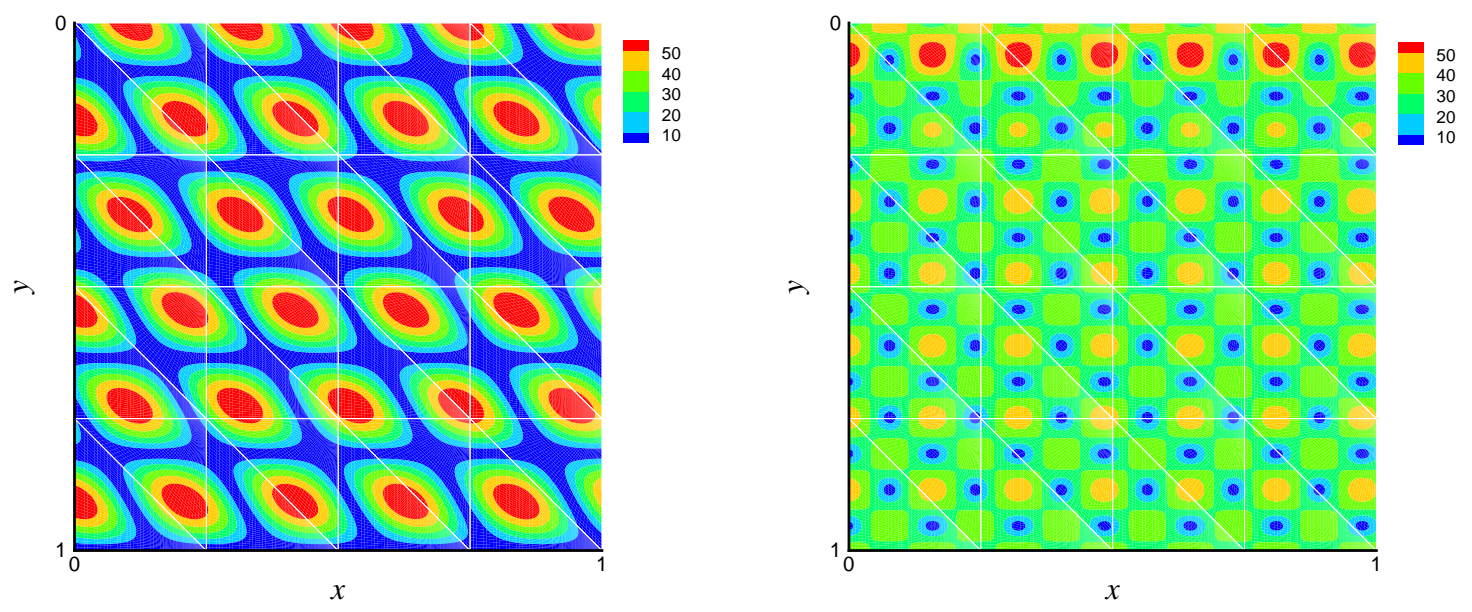

Figure 4: Real part of the displacement due to an SV-plane wave hitting a free surface of a semi-infinite elastic medium, $\omega=30, m_{P}=m_{S}=16$, (left) $\beta=60^{\circ}, \varepsilon_{2}=0.6 \%$, (right) $\beta=50^{\circ}, \varepsilon_{2}=0.6 \%$.

\subsection{Pure Rayleigh waves}

Rayleigh waves are present near the soil free surface and they decay exponentially with depth. They appear when body waves are reflected at the free surface. Part of their energy is converted into Rayleigh waves which propagate with their own wave number $k_{R}$ given by the 
approximate expression $k_{R} \approx k_{S} / 0.9194$, for the same considered material $(\lambda=\mu=\rho=1$ ). The horizontal and vertical components of the displacement field caused by the Rayleigh waves are given by the following expressions [10]

$$
\begin{aligned}
u & =\sqrt{k_{R}^{2}-k_{S}^{2}}\left[e^{-\sqrt{k_{R}^{2}-k_{S}^{2}} y}-\frac{2 k_{R}^{2}}{2 k_{R}^{2}-k_{S}^{2}} e^{-\sqrt{k_{R}^{2}-k_{P}^{2}} y}\right] e^{\mathrm{i} k_{R} x}, \\
v & =\mathrm{i} k_{R}\left[e^{-\sqrt{k_{R}^{2}-k_{S}^{2}} y}-\frac{2 \sqrt{\left(k_{R}^{2}-k_{P}^{2}\right)\left(k_{R}^{2}-k_{S}^{2}\right)}}{2 k_{R}^{2}-k_{S}^{2}} e^{-\sqrt{k_{R}^{2}-k_{P}^{2}} y}\right] e^{\mathrm{i} k_{R} x} .
\end{aligned}
$$

For the same computational domain of Figure 2, the Rayleigh induced displacements are imposed on its boundary and the model developed above is used to recover the displacement field inside the domain. It is worth pointing out again to the fact that Rayleigh waves propagate with the wave number $k_{R}$, while the plane wave approximation of expression (19) of the displacement field uses the pressure and shear wave numbers $k_{P}$ and $k_{S}$, rather than $k_{R}$. Figure 5 shows two examples of numerical tests involving the Rayleigh wave problem for frequencies $\omega=10$ and $\omega=20$. Both figures show the displacement profile which displays ellipses with major axes normal to the free surface. Moreover, the displacements occur mainly near the free surface and decay rapidly with depth. For $\omega=10$, only 16 plane waves are used to approximate the displacement field with $m_{P}=m_{S}=8$, leading to an error of $0.5 \%$. In the case of $\omega=20$, 28 plane waves are used such that $m_{P}=m_{S}=14$ and the $L_{2}$-error remains low as well, $\varepsilon_{2}=0.8 \%$. This clearly indicates the good performance of the proposed approximation and its ability to capture Rayleigh waves in spite of the model not explicitly including the wave number $k_{R}$ in the plane wave approximation (20).
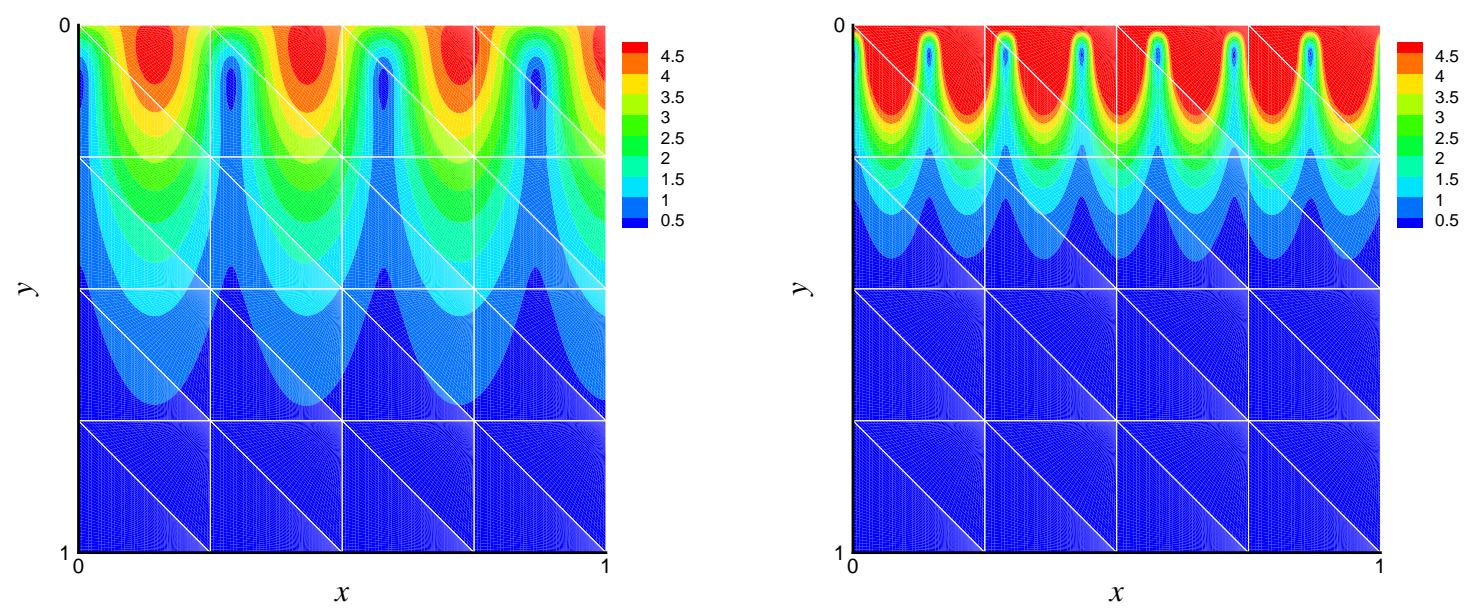

Figure 5: Real part of the displacement due to Rayleigh waves, (left) $\omega=10, m_{P}=m_{S}=8, \varepsilon_{2}=0.5 \%$, (right) $\omega=20, m_{P}=m_{S}=14, \varepsilon_{2}=0.8 \%$.

\subsection{SV plane wave hitting a plane interface}

In multilayered soils, for example, wave transmission and reflection occur at interfaces. In this sub-section, an incident elastic wave hitting a plane interface between two semi-infinite elastic media is considered. In general, this produces compression and shear waves in both media. Figure 6 shows a schematic diagram of an incident SV-plane wave hitting such interface. 
For this case, the compression wave potential is therefore given by the reflected and transmitted waves

$$
\phi=R_{P} e^{\mathrm{i} k(-x-y \tan \alpha)}+T_{P} e^{\mathrm{i} k(-x-y \tan \gamma)},
$$

while the shear wave potential is the sum of the incident, reflected and transmitted waves

$$
\psi=1 e^{\mathrm{i} k(-x+y \tan \beta)}+R_{S} e^{\mathrm{i} k(-x-y \tan \beta)}+T_{S} e^{\mathrm{i} k(-x-y \tan \theta)},
$$

The wave number $k$ is now given by $k=k_{1}^{P} \cos \alpha=k_{1}^{S} \cos \beta=k_{2}^{P} \cos \gamma=k_{2}^{S} \cos \theta$. The coefficients $R_{P}, R_{S}, T_{P}$ and $T_{S}$ are obtained by ensuring continuity of the displacement and stress fields at the interface [9]. The horizontal and vertical components of the displacement field, deduced from expressions (27), are imposed on the boundary of the computational domain of Figure 2 representing now two semi-infinite media in contact at the horizontal interface $y=$ 0.5. Two cases are considered where a soft layer overlies a hard layer and vice versa with a ratio of densities of $1 / 3$. In both cases, the $S V$ plane wave is incident in the lower layer with $\beta=60^{\circ}$ and $\omega=10$. A total of 24 plane waves are chosen such that $m_{P}=10$ and $m_{S}=14$. Figure 7 shows the numerical results of the real part of the displacement for both cases. The low $L_{2}$ errors, $0.8 \%$ and $0.7 \%$, show the ability of the developed model to simulate such problems while ensuring continuity at the interface thanks to the Lagrange multipliers.

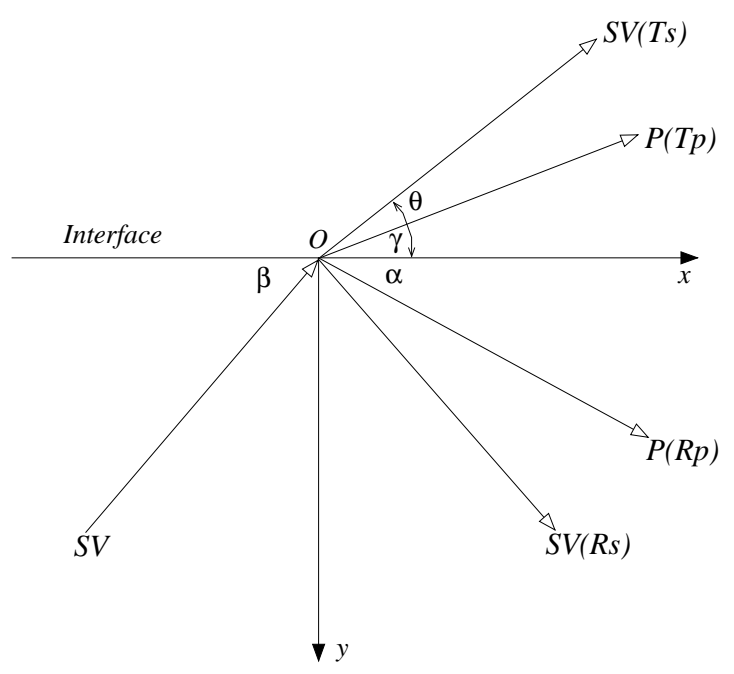

Figure 6: SV-plane wave hitting a plane interface between two semi-infinite elastic media. 

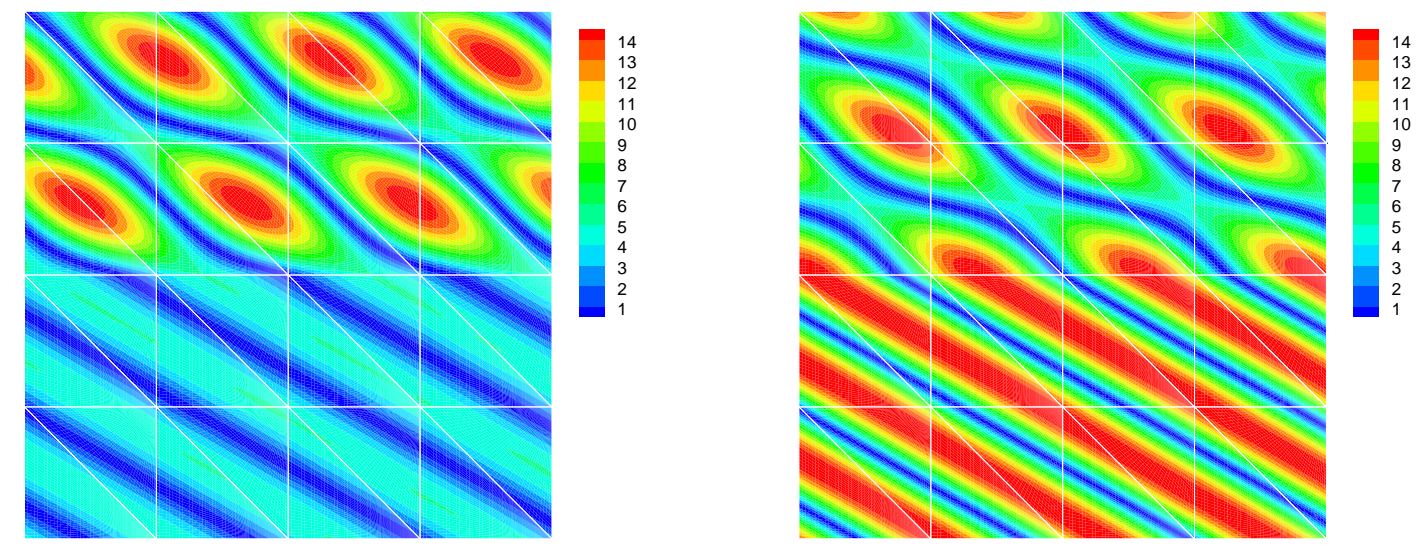

Figure 7: Real part of the displacement due to an SV-plane wave hitting a plane interface between two semi-infinite elastic media, $\omega=10, m_{P}=10, m_{S}=14, \beta=60^{\circ}$, (left) hard layer overlying soft layer $\varepsilon_{2}=0.8 \%$, (right) soft layer overlying hard layer $\varepsilon_{2}=0.7 \%$.

\section{CONCLUSIONS}

In this work, the PUFEM is extended to deal with wave problems in elastic layered media by incorporating Lagrange multipliers to ensure compatibility conditions at the interfaces between the layers. The model is validated by considering a simple example of an SV plane wave transmitted at the plane interface between two semi-infinite elastic media. The model is also shown to deal with Rayleigh wave propagation without explicitly including its wave number in the displacement approximation. It also simulates elastic wave problems in presence of evanescent modes.

The results presented above are preliminary. They provide an indication of the ability of the PUFEM model to deal with various elastic wave problems in homogeneous and inhomogeneous media. Robustness and Accuracy of this model are currently being assessed through the consideration of different parameters such as the mesh size, the number of plane wave basis in the approximation of the displacement field and in the Lagrange multiplier, the problem frequency and the significant variations in material properties between layers.

\section{Acknowledgements}

The authors are grateful to the EPSRC for funding this work under grant number EP/I018042/1.

\section{REFERENCES}

[1] A. El Kacimi, O. Laghrouche. Numerical modelling of elastic wave scattering in frequency domain by the Partition of Unity Finite Element Method. Int. J. Numer. Methods Engrg. 2009; 77:1646-1669.

[2] A. El Kacimi, O. Laghrouche. Improvement of PUFEM for the numerical solution of high frequency elastic wave scattering on unstructured triangular mesh grids. Int. J. Numer. Methods Engrg. 2010; 84:330-350. 
[3] A. El Kacimi, O. Laghrouche. Wavelet based ILU preconditioners for the numerical solution by PUFEM of high frequency elastic wave scattering. Journal of Computational Physics 2011; 230:3119-3134.

[4] R. Tezaur, L. Zhang, C. Farhat. A discontinous enrichmment method for capturing envanescent waves in multiscale fluid and fluid/solid problems. Comp. Meth. Appl. Mech. Engng. 2008; 197 1680-1698.

[5] T. Huttunen, Kaipio JP, Monk P. An ultra-weak method for acoustic fluid-solid interaction. J. Comput. Appl. Math. 2008; 213:166-185.

[6] E. Perrey-Debain, J. Trevelyan and P. Bettess. P-wave and S-wave decomposition in boundary integral equation for plane elastodynamic problems. Commun. Numer. Meth. Engng. 2003; 19:945-958.

[7] O. Laghrouche, P. Bettess, E. Perrey-Debain, J. Trevelyan. Wave interpolation finite elements for Helmholtz problems with jumps in the wave speed. Comput. Meth. Appl. Mech. Engng. 2005; 194:367-381.

[8] P. Bettess and J.A. Bettess. A profile matrix solver with built-in constraint facility. Eng. Comput. 1986; 3:209-216.

[9] W.M. Ewing, W.S. Jardetzky, F. Press. Elastic Waves in Layered Media. McGraw-Hill Book Company. New York, 1957.

[10] B.A. Auld. Acoustic Fields and Waves in Solids. John Wiley and Sons, 1973. 